Meta

Journal des traducteurs

Translators' Journal

\title{
Claves para una sociología de la traducción de narrativa a partir de COVALT (1990-2000)
}

\section{María Dolores Burdeus et Joan Verdegal}

Volume 50, numéro 4, décembre 2005

Pour une traductologie proactive - Actes

For a Proactive Translatology - Proceedings

Por una traductología proactiva - Actas

URI : https://id.erudit.org/iderudit/019832ar

DOI : https://doi.org/10.7202/019832ar

Aller au sommaire du numéro

Éditeur(s)

Les Presses de l'Université de Montréal

ISSN

0026-0452 (imprimé)

1492-1421 (numérique)

Découvrir la revue

Citer cet article

Burdeus, M. D. \& Verdegal, J. (2005). Claves para una sociología de la traducción de narrativa a partir de COVALT (1990-2000). Meta, 50(4).

https://doi.org/10.7202/019832ar
Résumé de l'article

Cet article se propose de montrer comment utiliser proactivement la recherche sociologique du fait littéraire. Faisant un bilan de la traduction littéraire entre 1990 et 2000, COVALT (Corpus Valencien de Littérature Traduite) assure des conclusions très utiles pour la traductologie, notamment pour la sociologie de la traduction, mais aussi pour des recherches visant sur des aspects empiriques concernant l'activité traductrice (en milieu professionnel), l'enseignement de la traduction (en milieu universitaire) et le processus de traduction. La gestion-exploitationintégrale d'un corpus de littérature traduite s'avère fondamentale pour mesurer objectivement les résultats des actes de traduction. 


\title{
Claves para una sociología de la traducción de narrativa a partir de COVALT (1990-2000) ${ }^{1}$
}

\author{
MARÍA DOLORES BuRdEUS \\ Universitat Jaume I, Castelló, España \\ burdeus@trad.uji.es \\ JOAN VERDEGAL \\ Universitat Jaume I, Castelló, España \\ verdegal@trad.uji.es
}

\begin{abstract}
RÉSUMÉ
Cet article se propose de montrer comment utiliser proactivement la recherche sociologique du fait littéraire. Faisant un bilan de la traduction littéraire entre 1990 et 2000, COVALT (Corpus Valencien de Littérature Traduite) assure des conclusions très utiles pour la traductologie, notamment pour la sociologie de la traduction, mais aussi pour des recherches visant sur des aspects empiriques concernant l'activité traductrice (en milieu professionnel), l'enseignement de la traduction (en milieu universitaire) et le processus de traduction. La gestion-exploitation intégrale d'un corpus de littérature traduite s'avère fondamentale pour mesurer objectivement les résultats des actes de traduction.
\end{abstract}

\begin{abstract}
This paper aims to show how sociological research on the literary fact can be used proactively. From a balance of literary translation between 1990 and 2000, COVALT (Valencian Corpus of Translated Literature) draws useful conclusions for translation studies, particulary for the sociology of translation, but also for any research into empirical aspects concerning the translating activity (in a professional environment), the teaching of translation (in a university environment) and the translation process. The integral management-explotation of a corpus of translated literature is considered fundamental to the objective measurement of the results of translation acts.
\end{abstract}

\section{MOTS-CLÉS/KEYWORDS}

Corpus Valencien de Littérature Traduite (COVALT), sociologie de la traduction, gestionexploitation de corpus, proactivité de corpus.

\section{Antecedentes y objetivos}

El principal objetivo de este artículo es presentar las posibilidades de investigación sociológica del hecho literario a partir de un corpus limitado de literatura traducida en contexto valenciano. El punto de partida se remonta al año 1999, momento en que un grupo de profesores de los departamentos de Traducción y Comunicación y de Filología Inglesa y Románica de la Universitat Jaume I confluyeron en unos intereses investigadores que pretendían relacionar la producción literaria en diversas lenguas y su traducción. El primer fruto de aquel germen fue el proyecto denominado Corpus de traducciones valencianas de literatura narrativa (castellano y catalán) entre 1990 y 2000: editoriales y traductores, que se desarrolló entre los años 2001 y 2002. Le siguió una nueva edición (para la etapa 2003-2005), centrada sobre todo en la confección de una base de datos que permitiese estudiar los fenómenos fraseológicos: Elaboración de bases 
de datos bilingües de unidades fraseológicas basadas en el corpus COVALT. Finalmente, y de forma paralela al anterior (para el período 2003-2006), está vigente un tercer proyecto que, con el título Unidades de traducción de tipo fraseológico: análisis basado en el corpus COVALT, complementa los anteriores ${ }^{2}$.

Una de las muchas perspectivas de estudio que ofrecen estas vías investigadoras es indagar en aspectos sociológicos ligados a la literatura y -más específicamente- a la traducción de literatura. Hay que decir que COVALT (Corpus Valenciano de Literatura Traducida) está compuesto exclusivamente por obras de género narrativo, en sentido amplio, de manera que se han descartado (de momento) los demás géneros. El motivo principal de esta selección ha sido la mayor abundancia de publicaciones de narrativa, así como la consideración -admitida por todo el mundo- de que este género es precisamente el más traducido, el más publicado, el más comprado y el más leído ${ }^{3}$. Esto supone una ventaja nada despreciable, ya que la acotación al contexto valenciano hace poco representativo un corpus de géneros menos presentes en nuestro (¿reducido?) mercado editorial ${ }^{4}$. Por otra parte, el hecho de elegir un corpus de literatura traducida presenta una ventaja digna de tenerse en cuenta: la calidad de sus textos. Ciertamente, son muchos los investigadores de lingüística de corpus que se ven obligados a establecer filtros diversos que impidan o minimicen la irrupción de incorrecciones o de elementos no genuinos de las lenguas tratadas, en especial cuando sus fuentes proceden de Internet ${ }^{5}$. En contrapartida, los textos literarios (Marco, Verdegal, Hurtado, 1999: 167-168) ofrecen «una integración entre forma y contenido mayor de la habitual, y una especial vocación de originalidad», y, además,

\footnotetext{
Las obras literarias tienen la particularidad de poder combinar cualquier tipo de texto: narrativos, descriptivos, conceptuales, argumentativos, instructivos. Pueden producirse cambios de campo, ya que los textos literarios son híbridos en cuanto a los temas que tratan y pueden incluso albergar lenguajes de especialidad; cambios de tono, que reflejan las diferentes relaciones entre personajes, o entre narrador, personajes y público; cambios de modo, como la alternancia (en los narrativos) entre la narración propiamente dicha y el diálogo, que presuponen un medio oral. Pueden aparecer diversos estilos, diferentes dialectos sociales, geográficos, temporales, idiolectos particulares, con valores canónicos (es decir, inmutables y acuñados en cuanto a la forma). Además, suelen estar anclados en la cultura de partida y presentar, por lo tanto, múltiples referencias culturales.
}

Si partimos del principio de que, en traducción, se consideran básicamente tres tipos de córpora ( $\mathrm{a} /$ los monolingües, formados por textos redactados en lengua de llegada; $\mathrm{b} /$ los bilingües comparables, con textos de partida en dos idiomas, y c/ los paralelos, con textos de partida en un idioma y sus traducciones a otro idioma), resultará fácil entender por qué nos han interesado precisamente desde un principio los paralelos.

Antes de comenzar la tarea descriptiva que aquí nos ocupa, será necesario presentar las líneas básicas del método utilizado, ya que la dispersión de los criterios que tenemos a nuestro alcance podría dificultar los avances. Queremos referirnos a la sociología de la literatura y a la sociología literaria (Leenhardt, 2003). Si bien a primera vista pueden parecer subdisciplinas semejantes, hay que señalar que sus enfoques son diferentes. La sociología de la literatura, como parte integrante de la sociología, intenta aplicar sus métodos a la difusión, al éxito, a los públicos receptores, a la institución literaria y a los grupos profesionales (por ejemplo los escritores, profesores o críticos), es decir, a todo aquello que, en literatura, no es propiamente texto. Por el contrario, la sociología literaria es considerada uno de los métodos de las ciencias literarias, orientado hacia el texto y con el objetivo de facilitar su comprensión. Pues bien, la presente aportación intenta cubrir lagunas propias de la primera subdisciplina, dejando para etapas posteriores conclusiones procedentes del segundo enfoque. Ambas orientaciones, perfectamente armonizadas, nos ayudarán a la larga a conocer mejor la respuesta a ciertas preguntas constantemente formuladas ${ }^{6}:$ ¿qué se ha traducido?, ¿cuándo se ha traducido?, ¿cómo se ha 
traducido?, ¿quién ha traducido?, ¿cuánto se ha traducido?, ¿dónde se ha traducido?, ¿por qué se ha traducido?, ¿de dónde se ha traducido?...

\section{Ordenar para comprender}

Una de las vías de investigación que ofrece la sociología de la literatura es la recepción (Warning, 1989; Guzman, 1995), entendida en este caso como el estudio de los impactos de las obras literarias (y de sus autores) en un momento dado de la historia (factor sincrónico) y en una o varias de las lenguas y contextos sociales a los que ha sido traducida, pero soslayando (de momento) aspectos ideológicos e institucionales que desviarían la atención primaria del analista. La investigación que aquí se presenta puede considerarse de tipo observacional (en el sentido en que lo describe Gile, 1998), y sienta sus bases en la ordenación de los datos disponibles y pertinentes; esta tarea previa (nada fácil y siempre costosa en tiempo) es la mejor garantía de objetividad. Para ello, se ha procedido al etiquetado de COVALT eligiendo una marcación estructural parcial, basada sólo en aquellos datos pertenecientes al universo externo a los textos, es decir, en las filiaciones de las obras que componen el corpus. Las referencias proceden del vaciado de dos bases de datos del Ministerio de Cultura español: el Catálogo de las Bibliotecas Públicas del Estado (BPE) y el de la Agencia del ISBN. El primero de los pasos ha consistido en recopilar toda esa información en una base de datos de formato Microsoft Access, de forma que cada columna corresponde a una etiqueta distinta ${ }^{7}$. El orden lógico que se propone es el siguiente:

- Columna 1: Número de orden

- Columna 2: Número del autor/a

- Columna 3: Número de la obra

- Columna 4: Autor/a y fechas de nacimiento y de defunción

- Columna 5: Título de la obra original

- Columna 6: Título de la obra traducida

- Columna 7: Fecha de publicación de la obra original y lengua

- Columna 8: Fecha de publicación de la traducción y lengua

- Columna 9: Diferencia temporal entre ambas fechas

- Columna 10: Traductor/a

- Columna 11: Subgénero

- Columna 12: Editorial en que se publicó el original

- Columna 13: Editorial en que se publicó la traducción

La primera ventaja que obtenemos con ello es una notable versatilidad en el procesamiento de los datos. En efecto, pues a la mera ordenación numérica (que nos permite contabilizar el total de obras y autores que constituyen el corpus a estudiar) se añade la transparencia de la cuarta columna, en la que aparece la cronología vital de los autores. Este detalle no resulta en absoluto superfluo, ya que delimita el contexto de la obra original, facilitando así la ubicación de su traducción; ambos datos (columnas 7 y 8) servirán para calcular la distancia temporal entre la primera y la segunda circunstancia (columna 9), lo que puede ser útil, por ejemplo, para deducir la pervivencia de la obra (si se tienen en cuenta las posibles traducciones anteriores) o para desvelar el interés de la editorial por encargar su traducción (no es lo mismo apostar por un autor consagrado por el que no hay que abonar derechos de traducción -si han transcurrido ochenta años- que hacerlo por un autor contemporáneo -lo que implica comprar los derechos a las editoriales originales $)^{8}$. Las columnas 5 y 6 ofrecen otras posibilidades de análisis: ¿se ha traducido literalmente el título?, ¿se ha seguido otro criterio? En este caso, ¿cuál ha sido el motivo? Las respuestas a esas 
preguntas no son banales, ya que nos pueden informar de aspectos pragmáticos adoptados por los traductores o de motivaciones comerciales nada despreciables para los intereses de las editoriales, como la de publicar sólo una parte de la obra original dándole la categoría de obra completa. A partir de las columnas 7 y 8 (además de la distancia cronológica anotada en la columna 9) puede inferirse la trayectoria traductora, es decir, el número total de obras originales de una determinada lengua vertidas a la lengua o a las lenguas del territorio geográfico analizado; así, podrá determinarse una prelación de las editoriales (consideradas globalmente o individualmente) por ciertas fuentes literarias en detrimento de otras (aspecto de cierto interés si se quieren conocer las políticas editoriales). La columna 10 puede ser reveladora del número de traductores que han intervenido en ese contexto editorial, y ello hasta tal punto que podemos deducir su adscripción a una u otra editorial, su volumen de trabajo, los subgéneros que ha abordado o su lengua o lenguas de partida y de llegada. Tener a la vista precisamente los subgéneros narrativos (columna 11) nos ofrece igualmente ciertas posibilidades de estudio: a partir de la clasificación por materias que aparece en la ficha del ISBN, podemos desvelar que ciertos autores publican determinado subgénero, o que otros quedan englobados o encasillados en otro, o que algunas lenguas suministran más material de cierto subgénero que las demás. La información que puede procesarse de las columnas 12 y 13 resulta de mayor utilidad en el caso de obras contemporáneas y de editoriales que coexisten en el tiempo, ya que ello permite descubrir algunos emparejamientos reiterados (fruto, sin duda, de una estrecha colaboración entre editoriales de textos originales y editoriales traductoras), pero también cierta competitividad entre editoriales (que, a veces, publican casi simultáneamente las mismas traducciones, encargadas a traductores diferentes).

La segunda ventaja que ofrece la base de datos es su ductilidad, es decir, las posibilidades que tiene de presentarse en diferentes formatos. En la medida en que nos interese un recuento de editoriales, procederemos sin más a ordenar las dos últimas columnas; si lo que nos conviene es contabilizar los subgéneros, haremos lo propio con la columna 11; si queremos tener presente el volumen de trabajo de los traductores, ordenaremos la columna 10; si pretendemos desvelar qué obras se han traducido con mayor celeridad, ordenaremos la columna 9, y así sucesivamente con los demás datos procesados.

\section{Primeras conclusiones parciales}

\subsection{Los traductores}

Las 211 entradas que componen la base de datos de literatura traducida en contexto valenciano (entre 1990 y 2000) se convierten en 204 si restamos las que aparecen por duplicado, es decir, las que han sido publicadas por dos editoriales diferentes ${ }^{9}$. Esos libros son los siguientes:

1) CAlleja PÉRez, Seve (1953-): Per què a Mari José li diuen José Mari? (1997: Bromera; 1997: Tàndem). Traductora compartida: Blanca Cassany.

2) CASALDERrey, Fina (1951-): Esc, quin fàstic (1997: Bromera; 1997: Tàndem). Traductor compartido: Avel·lí Llimac.

3) CASARES, Carlos (1941-2002): La bicicleta de Lolo (1996: Bromera; 1996: Tàndem). Traductor compartido: Avel-lí Llimac.

4) IGERABIDE, Juan Kruz (1956-): Somiatruites (1996: Bromera; 1996: Tàndem). Traductor compartido: Avel·lí Llimac.

5) Keselman Porter, Gabriela (1953-): Per què? (1996: Bromera; 1996: Tàndem). Traductora compartida: Rosa Serrano.

6) LondON, Jack (1876-1916): La crida del bosc (1995: Germania); La crida salvatge (1998: Bromera) Diferentes traductores: Tomasa Plata; Joan E. Pellicer e Inés Fernández. 
7) Mendiguren Elizegi, Xabier (1964-): Per què no canta el pit-roig? (1997: Bromera; 1997: Tàndem). Traductora compartida: Blanca Cassany.

8) Prestifilippo Lajud-Cura, Pablo Rafael (1960-): Paraules màgiques (1997: Tàndem; 1998: Bromera). Traductor compartido: Daniel Rozados.

Queda de manifiesto que ha sido habitual entre editoriales compartir el trabajo del traductor: aquí se da en 7 de las 8 publicaciones ${ }^{10}$. Sin embargo, esa estrategia se ha desarrollado exclusivamente a lo largo de tres años (entre 1996 y 1998), y sólo entre las otras lenguas de España y el catalán, implicando a cuatro traductores. Esa política editorial de compartir la labor traductora permite, además de reducir los costes, compensar incluso que aparezcan simultáneamente en el mercado dos libros con el mismo título. La única excepción se produce en el libro de Jack London The Call of the Wild, cuando el desfase de tres años entre 1995 y 1998 provoca incluso dos versiones diferentes para el título traducido: La crida del bosc (publicada en la editorial Germania) da paso a La crida salvatge (en Bromera).

En esos 204 libros que componen el corpus han intervenido 126 traductores (92 hombres y 34 mujeres) ${ }^{11}$, aunque no siempre en solitario, ya que en 16 ocasiones han trabajado en parejas y una vez en cuarteto. Esas parejas han sido hombre-hombre en 5 ocasiones, hombremujer en 10 ocasiones, y mujer-mujer sólo una vez. La mayoría de los traductores (individualmente o en pareja) sólo aportan un trabajo en ese período: son 88. De entre los restantes (que son 38), 16 han realizado 2 traducciones, 7 han traducido 3 veces, 4 han traducido 4 veces, 2 lo han hecho 5 veces, 3 han realizado 6 traducciones, 3 han traducido 7 veces y 1 ha traducido 17 libros. Si bien lo habitual es traducir de una única lengua, no es así en el caso de 12 traductores. Podemos observar esos detalles en la siguiente tabla, donde se recogen sus nombres y el volumen de traducciones de cada par de lenguas:

\begin{tabular}{|c|c|c|c|c|c|c|c|c|}
\hline & ing >cat & fra>cat & ale $>$ cat & por>cat & ita>cat & esp>cat & eus $>$ cat & gal>cat \\
\hline D. Ardit & 1 & 1 & & & & & & \\
\hline J.C. Asins & & 2 & & & & 1 & & \\
\hline F. Boada & & & & 1 & & & 1 & \\
\hline E. Casasses & 3 & 2 & & & & & & \\
\hline B. Cassany & & & & & & 1 & 2 & \\
\hline J. Cortés & 1 & 2 & & & & & & \\
\hline J. Franco & 8 & 1 & 1 & & & 1 & & 6 \\
\hline A. Llimac & & & & & & & 1 & 3 \\
\hline S. Pallarés & & & & & 1 & & & 2 \\
\hline $\begin{array}{l}\text { M. } \\
\text { Rodríguez }\end{array}$ & 1 & 1 & & & & & & \\
\hline R. Serrano & & 3 & & & & 2 & & \\
\hline E. Sòria & 1 & & 1 & & & & & \\
\hline TOTAL & 15 & 12 & 2 & 1 & 1 & 5 & 4 & 11 \\
\hline
\end{tabular}

La conclusión más evidente que se deduce de estos datos es que los traductores de este período (1990-2000) se han especializado en una de las dos lenguas de la Comunidad Autónoma (el catalán sobre todo), ya que nunca traducen hacia las dos. Cinco de los traductores de la tabla anterior compaginan el inglés y el francés, con un total de 21 obras, y sólo uno de ellos hace extensiva su labor traductora a otras tres lenguas; es el caso de Josep Franco, que también traduce del alemán, del español y del gallego. Resulta significativo constatar que las editoriales para quienes trabajan esos "politraductores" son normalmente las más productivas: Bromera, Eliseu Climent y Tàndem (véase el apartado 3.4).

Otro aspecto a considerar es la adscripción de los traductores a las diferentes editoriales (columnas 10 y 13 de COVALT). El resultado que se observa a simple vista, al cotejar las adscripciones de los traductores que han traducido más de una obra, es una notable fidelidad a 
sus editoriales. En efecto, de los 38 profesionales identificados, 26 traducen en exclusiva para una única editorial, y sólo 12 diversifican sus fuentes de trabajo. Podríamos saber la causa de esas fidelidades o infidelidades entrevistando a los traductores (aun a riesgo de recibir respuestas subjetivas o sesgadas), pero la mera observación de las entradas nos ofrece algunas pistas. En 4 de esos 12 casos el motivo es bastante transparente, ya que se trata de los traductores que han compartido una o más de sus traducciones para dos editoriales; en cuanto a las demás infidelidades (que son 8), las consideraremos meramente coyunturales.

\subsection{Los autores}

De entre los 158 autores publicados en ese período de once años, los más prolíficos han sido los siguientes: Jack London, con 6 traducciones (pero que corresponden a 5 originales, ya que la traducción de The Call of the Wild ha sido publicada por dos editoriales), Edgar A. Poe y Robert L. Stevenson (con 5 publicaciones de sus originales) y A.C. Doyle (con cuatro libros traducidos). Tanto estos como los siguientes más productivos se presentan en esta lista (indicamos entre [ ] si se da una obra repetida):

\begin{tabular}{|c|c|c|c|c|c|c|}
\hline \multirow{2}{*}{$\begin{array}{l}\begin{array}{l}\text { Autor con } 6 \\
\text { traducciones }\end{array} \\
\text { London }\end{array}$} & \multirow{2}{*}{$\begin{array}{l}\begin{array}{l}\text { Autores con } 5 \\
\text { traducciones }\end{array} \\
\text { Poe } \\
\text { Stevenson }\end{array}$} & \multirow{2}{*}{$\begin{array}{l}\begin{array}{l}\text { Autores con } 4 \\
\text { traducciones }\end{array} \\
\text { Doyle }\end{array}$} & Autores con 3 & \multicolumn{2}{|c|}{ Autores con 2 traducciones } & \multirow{2}{*}{$\begin{array}{l}\begin{array}{l}\text { Autores con } 1 \\
\text { trad. repetida }\end{array} \\
\text { S. Calleja } \\
{[1+1]} \\
\text { Keselman } \\
{[1+1]} \\
\text { Prestifilippo } \\
{[1+1]}\end{array}$} \\
\hline & & & $\begin{array}{l}\text { Atxaga } \\
\text { Casalderrey } \\
{[3+1]} \\
\text { Melville } \\
\text { Mendiguren } \\
{[3+1]} \\
\text { Wilde } \\
\text { Wilson }\end{array}$ & $\begin{array}{l}\text { Blasco Ibáñez } \\
\text { Casares [2+1] } \\
\text { Conrad } \\
\text { Docampo } \\
\text { Fernández Paz } \\
\text { Gautier } \\
\text { Igerabide } \\
\text { [2+1] } \\
\text { Lawrence } \\
\text { Macfarlane/ } \\
\text { MacPherson }\end{array}$ & $\begin{array}{l}\text { Maupassant } \\
\text { Kafka } \\
\text { Pennac } \\
\text { Sommer- } \\
\text { Bodenburg } \\
\text { Stendhal } \\
\text { Troyes } \\
\text { Voltaire } \\
\text { Xukri } \\
\text { Zubizarreta }\end{array}$ & \\
\hline
\end{tabular}

Se trata de 31 autores (o coautores) que abarcan 77 de las 204 traducciones (37,74\% del total). El resto lo constituye un grupo numeroso de 127 autores que sólo han visto publicada una traducción de una de sus obras. La tabla nos descubre que los cuatro autores que se encuentran en cabeza han escrito en inglés, así como tres del grupo siguiente, lo que nos obliga a preguntarnos sobre las lenguas de las traducciones.

\subsection{Las lenguas, los subgéneros y los destinatarios}

Otro de los recursos que ayudará a comprender los parámetros de recepción en el período estudiado es el análisis del volumen de obras originales por lenguas. Ese recuento nos permite descubrir su porcentaje de presencia en las librerías. De las 204 obras diferentes traducidas (ya hemos descontado las 7 repetidas), 73 proceden del inglés ${ }^{12}$, 49 del francés, 19 del español ${ }^{13}$, 15 del alemán, 14 del gallego ${ }^{14}$, 10 del euskera, 10 del italiano, 5 del árabe, 4 del portugués, 2 del griego clásico, 1 del griego moderno, 1 del latín y 1 del finlandés. En definitiva, las combinaciones lingüísticas que se dan en COVALT son las que se indican a continuación, con expresión del número de obras y de su porcentaje respecto al total. Se presentan en tablas diferentes los libros traducidos al catalán (por su carácter mayoritario) y los traducidos al español. No se recogen las diversas ediciones procedentes de una misma traducción. 
LO>catalán $(84,31 \%)$

\begin{tabular}{|c|c|c|c|c|c|c|c|c|c|c|c|c|c|}
\hline $\begin{array}{l}\text { ing> } \\
\text { cat }\end{array}$ & $\begin{array}{l}\text { fra> } \\
\text { cat }\end{array}$ & $\begin{array}{l}\text { esp> } \\
\text { cat }\end{array}$ & $\begin{array}{l}\text { gal> } \\
\text { cat }\end{array}$ & $\begin{array}{l}\text { ale> } \\
\text { cat }\end{array}$ & $\begin{array}{l}\text { eus> } \\
\text { cat }\end{array}$ & $\begin{array}{l}\text { ita> } \\
\text { cat }\end{array}$ & $\begin{array}{l}\text { ára> } \\
\text { cat }\end{array}$ & $\begin{array}{l}\text { por> } \\
\text { cat }\end{array}$ & $\begin{array}{l}\text { grc> } \\
\text { cat }\end{array}$ & $\begin{array}{l}\text { grm> } \\
\text { cat }\end{array}$ & $\begin{array}{l}\text { lat> } \\
\text { cat }\end{array}$ & $\begin{array}{l}\text { fin> } \\
\text { cat }\end{array}$ & TOTAL \\
\hline 59 & 36 & 19 & 14 & 12 & 10 & 9 & 4 & 4 & 2 & 1 & 1 & 1 & 172 \\
\hline 34,30 & 20,93 & 11,04 & 8,13 & 6,97 & 5,81 & 5,23 & 2,32 & 2,32 & 1,16 & 0,58 & 0,58 & 0,58 & $100 \%$ \\
\hline
\end{tabular}

LO>español $(15,68 \%)$

\begin{tabular}{|l|l|l|l|l|l|l|l|l|l|l|l|l|l|}
\hline $\begin{array}{l}\text { ing }> \\
\text { esp }\end{array}$ & $\begin{array}{l}\text { fra }> \\
\text { esp }\end{array}$ & $\begin{array}{l}\text { cat> } \\
\text { esp }\end{array}$ & $\begin{array}{l}\text { gal> } \\
\text { esp }\end{array}$ & $\begin{array}{l}\text { ale> } \\
\text { esp }\end{array}$ & $\begin{array}{l}\text { eus> } \\
\text { esp }\end{array}$ & $\begin{array}{l}\text { ita> } \\
\text { esp }\end{array}$ & $\begin{array}{l}\text { ára> } \\
\text { esp }\end{array}$ & $\begin{array}{l}\text { por> } \\
\text { esp }\end{array}$ & $\begin{array}{l}\text { grc> } \\
\text { esp }\end{array}$ & $\begin{array}{l}\text { grm> } \\
\text { esp }\end{array}$ & $\begin{array}{l}\text { lat> } \\
\text { esp }\end{array}$ & $\begin{array}{l}\text { fin> } \\
\text { esp }\end{array}$ & \begin{tabular}{l} 
TOTAL \\
\hline 14
\end{tabular} \\
\hline 13 & & & 3 & & 1 & 1 & & & & & & 32 \\
\hline 43,75 & 40,62 & & & 9,37 & & 3,12 & 3,12 & & & & & & $100 \%$ \\
\hline
\end{tabular}

Con los datos de que disponemos, corresponde ahora determinar si existe alguna relación entre la lengua de origen y el subgénero publicado. Siguiendo las mismas pautas observacionales, no resultará difícil deducir qué tipo de literatura reciben los lectores de traducciones de una u otra lengua, y si ello sigue unos criterios determinados aplicados por las editoriales. A partir de la ficha catalográfica del ISBN, entresacamos del apartado "Materias" los subgéneros en prosa que han cultivado los autores. Ello nos obliga a clasificar por orden alfabético esos subgéneros, junto a los cuales anotamos el número de obras adscritas a cada uno de ellos (debemos tener presente que a muchas de ellas se les asigna una combinación de materias):

B (Biografías): 1

DP (Derecho penal): 1

HC (Historia y crítica): 17

LEP (Literatura licenciosa, erótica y pornográfica): 3

LG (Literatura general): 1

LIJ (Literatura infantil y juvenil): 40

LIJ-HC (Literatura infantil y juvenil. Historia y crítica): 1

LIJ-NC (Literatura infantil y juvenil. Novela y cuento): 34

NC (Novela y cuento): 95

NC-HC (Novela y cuento. Historia y crítica): 1

OG (Otros géneros): 6

OL (Otras literaturas): 10

OT-DC (Organización del trabajo. Divulgación de la ciencia): 1

Así pues, esas 204 obras diferentes (en realidad 211 publicaciones) responden a unos subgéneros que, si bien no son del todo estrictos en su asignación ${ }^{15}$, nos pueden ilustrar de su presencia en la etapa estudiada. Si la lista anterior se traslada a una tabla ordenada por índice de frecuencia, obtenemos lo siguiente:

\begin{tabular}{|l|l|l|l|l|l|l|l|l|l|l|l|l|l|}
\hline $\mathbf{N C}$ & $\begin{array}{l}\text { LIJ- } \\
\text { NC }\end{array}$ & LIJ & HC & OL & OG & LEP & $\mathbf{B}$ & DP & LG & $\begin{array}{l}\text { LIJ- } \\
\text { HC }\end{array}$ & $\begin{array}{l}\text { NC- } \\
\text { HC }\end{array}$ & $\begin{array}{l}\text { OT- } \\
\text { DC }\end{array}$ & TOTAL \\
\hline 95 & 34 & 33 & 17 & 10 & 6 & 3 & 1 & 1 & 1 & 1 & 1 & 1 & 204 \\
\hline $\begin{array}{l}46,5 \\
6\end{array}$ & $\begin{array}{l}16,6 \\
6\end{array}$ & 16,1 & 8,33 & 4,90 & 2,94 & 1,47 & 0,49 & 0,49 & 0,49 & 0,49 & 0,49 & 0,49 & $100 \%$ \\
\hline
\end{tabular}

Con esta visión de conjunto podemos ya comprobar el papel relevante de los libros dirigidos a un público joven, contenidos en los epígrafes LIJ-NC, LIJ y LIJ-HC (con 68 obras), a los que se podría añadir una veintena de títulos que, aunque clasificados como NC, tienen como destinatarios también a lectores jóvenes, como lo atestiguan sus formatos y las colecciones en las que se publican, que incluyen una explotación didáctica y cuestionarios de lectura. Ese bloque estaría representado por unas 90 obras, es decir, el 44,11\% de la producción 
total. Ello significa, a todas luces, que las editoriales valencianas han apostado claramente entre 1990 y 2000 por un público en edad escolar (primaria y secundaria), lector potencial de este tipo de lectura de narrativa, tutorizada en parte por el personal docente de colegios e institutos, con el objetivo de incentivar la lectura de obras procedentes de otras literaturas, ya sean extranjeras o españolas. Por otra parte, la clasificación en subgéneros narrativos puede servir para restringir el corpus COVALT en subcórpora diferentes, con objeto - por ejemplo- de analizar aparte la literatura estrictamente infantil de la novela propiamente dicha ${ }^{16}$.

La combinación de las dos últimas tablas (la de las lenguas y la de los subgéneros) permite otras valoraciones, que complementan las anteriores.

\begin{tabular}{|l|l|l|l|l|l|l|l|l|l|l|l|l|l|l|}
\hline Subgéneros & ing & fra & esp & gal & eus & ale & ita & ára & por & grc & grm & lat & fin & TOTAL \\
\hline NC & 44 & 31 & 5 & 2 & 2 & 7 & 4 & & & & & & & 95 \\
\hline LIJ-NC & 13 & 4 & 11 & 4 & 1 & 1 & & & & & & & & 34 \\
\hline LIJ & 7 & 4 & 2 & 8 & 7 & 2 & 1 & & 2 & & & & & 33 \\
\hline HC & 4 & 7 & & & & 1 & & 1 & 1 & 2 & & 1 & & 17 \\
\hline OL & & & & & & & 4 & 3 & 1 & & 1 & & 1 & 10 \\
\hline OG & 3 & 2 & & & & 1 & & & & & & & & 6 \\
\hline LEP & 1 & & & & & 2 & & & & & & & & 3 \\
\hline OT-DC & & & 1 & & & & & & & & & & & 1 \\
\hline B & & & & & & & 1 & & & & & & & 1 \\
\hline DP & & & & & & 1 & & & & & & & & 1 \\
\hline LG & & 1 & & & & & & & & & & & & 1 \\
\hline LIJ-HC & 1 & & & & & & & & & & & & & 1 \\
\hline NC-HC & & & & & & & & 1 & & & & & & 1 \\
\hline TOTAL & 73 & 49 & 19 & 14 & 10 & 15 & 10 & 5 & 4 & 2 & 1 & 1 & 1 & 204 \\
\hline
\end{tabular}

Efectivamente, queda de manifiesto la importancia de la literatura dedicada a lectores jóvenes (recogida sobre todo en las tres filas LIJ-NC, LIJ y LIJ-HC), con porcentajes muy significativos según las lenguas:

\begin{tabular}{|l|l|l|l|l|l|l|l|l|l|l|l|l|l|}
\hline & ing & fra & esp & gal & eus & ale & ita & ára & por & grc & grm & lat & fin \\
\hline & 21 & 8 & 13 & 12 & 8 & 3 & 1 & & 2 & & & & \\
\hline$\%$ & 28,76 & 16,66 & 68,42 & 85,71 & 80 & 18,75 & 10,00 & & 50,00 & & & & \\
\hline
\end{tabular}

Este resultado nos confirma unos elevados porcentajes de ese tipo de literatura en dos de las lenguas (85,71\% en gallego y $80 \%$ en euskera), cuya literatura traducida es casi toda para ese tipo de público. Esa tendencia queda confirmada significativamente con las otras dos lenguas de la Península Ibérica (el español y el portugués), con porcentajes igualmente importantes (68,42 y 50\% respectivamente). En el caso del inglés, alemán y francés, los porcentajes de literatura para jóvenes se completan con colecciones incluidas en el subgénero NC. La literatura italiana no parece tener mucho hueco en ese contexto y, además, las de origen árabe, griego (sea clásico o moderno), latino y finlandés no publican ningún libro de ese tipo. Al respecto, es de destacar que es habitual entre editoriales españolas mantener convenios de intercambio de originales para su traducción, de forma que se ha constituido un mercado del libro "a cuatro bandas" entre el español, el catalán, el gallego y el euskera. La extrapolación de esas mismas conclusiones a un contexto cronológico más amplio nos permitiría conocer a fondo otros criterios editoriales que suelen quedar al margen de los estudios sociológicos relacionados con la publicación de literatura (Verdegal, 1997, 1999a, 1999b). 


\subsection{Las editoriales}

Son 20 las editoriales que desarrollaron su labor en ese período: 7 i Mig Edicions, Alcodre Ediciones, Amós Belinchón Editor, Edicions Bromera, Col-lectiu per l'ús de la llengua, Eliseu Climent Editor, Foro Ediciones, Editorial Germania, Instituto de Estudios Modernistas, Editorial La Máscara, Marjal-Grupo Edebé, Mateu Editors, Editorial MCA, Media Vaca, Numa Ediciones, Editorial Pre-Textos, Rotgle Edicions, Tabarca Llibres, Tàndem Edicions y Universidad de Valencia. En una ocasión, además, ha habido coedición de un libro entre Bromera y la Universidad de Valencia. En la tabla siguiente se expresa su ubicación geográfica, su volumen de producción editorial, los subgéneros abordados (con expresión de la cantidad en cada uno), las lenguas traducidas y las combinaciones lingüísticas.

\begin{tabular}{|c|c|c|c|c|c|c|}
\hline Editorial & Ciudad & Libros & Subgéneros & LO>cat & LO>esp & Combinaciones \\
\hline 7 i Mig & Alzira & 1 & $\mathrm{NC}(1)$ & 1 & & ita $>$ cat $=1$ \\
\hline Alcodre & Alicante & 1 & $\mathrm{NC}(1)$ & & 1 & in $g>\operatorname{esp}=1$ \\
\hline Amós Belinchón & Piporta & 3 & $\mathrm{NC}(1), \mathrm{LIJ}-\mathrm{NC}(1), \mathrm{OL}(1)$ & 3 & & $\begin{array}{l}\text { ale }>\text { cat }=1 \\
\text { ing }>\text { cat }=1 \\
\text { por }>\text { cat }=1\end{array}$ \\
\hline Bromera & Alzira & 91 & $\begin{array}{l}\text { NC (40), LIJ-NC (19), LIJ } \\
(19), \text { HC (7), OL (3), OG } \\
(2), \text { LIJ-HC (1) }\end{array}$ & 91 & & $\begin{array}{l}\text { ing }>\text { cat }=37 \\
\text { fra }>\text { cat }=21 \\
\text { gal }>\text { cat }=13 \\
\text { eus }>\text { cat }=7 \\
\text { ale }>\text { cat }=5 \\
\text { ára }>\text { cat }=4 \\
\text { esp }>\text { cat }=2 \\
\text { ita }>\text { cat }=1 \\
\text { por }>\text { cat }=1\end{array}$ \\
\hline $\begin{array}{l}\text { Bromera-Univ. } \\
\text { Valencia }\end{array}$ & $\begin{array}{l}\text { Alzira- } \\
\text { Valencia }\end{array}$ & 1 & OT-DC (1) & 1 & & esp $>$ cat $=1$ \\
\hline Col·lectiu P.L.L. & Silla & 1 & $\mathrm{NC}(1)$ & 1 & & esp $>$ cat $=1$ \\
\hline Eliseu Climent & Valencia & 35 & $\begin{array}{l}\text { NC (16), HC (8), OL (6), } \\
\text { LIJ (3), B (1), LIJ-NC (1) }\end{array}$ & 35 & & $\begin{array}{l}\text { ing }>\text { cat }=12 \\
\text { ita }>\text { cat }=7 \\
\text { fra }>\text { cat }=6 \\
\text { ale }>\text { cat }=3 \\
\text { grc }>\text { cat }=2 \\
\text { grm }>\text { cat }=1 \\
\text { esp }>\text { cat }=1 \\
\text { fin }>\text { cat }=1 \\
\text { lat }>\text { cat }=1 \\
\text { por }>\text { cat }=1\end{array}$ \\
\hline Foro & Valencia & 1 & $\mathrm{HC}(1)$ & & 1 & fra $>\operatorname{esp}=1$ \\
\hline Germania & Alzira & 7 & NC (5), OG (1), LIJ (1) & 7 & & $\begin{array}{l}\text { ing }>\text { cat }=4 \\
\text { fra }>\text { cat }=2 \\
\text { ale }>\text { cat }=1\end{array}$ \\
\hline I. E. Modernistas & Valencia & 2 & $\mathrm{NC}(2)$ & & 2 & fra $>\operatorname{esp}=2$ \\
\hline La Máscara & Valencia & 8 & $\begin{array}{l}\text { LEP (3), OG (3), LG (1), } \\
\text { NC (1) }\end{array}$ & & 8 & $\begin{array}{l}\text { ing }>\operatorname{esp}=4 \\
\text { fra }>\operatorname{esp}=2 \\
\text { ale }>\operatorname{esp}=2\end{array}$ \\
\hline Marjal & Mislata & 9 & LIJ-NC (9) & 9 & & esp $>$ cat $=9$ \\
\hline Mateu & Xàtiva & 1 & NC-HC (1) & & 1 & ára $>$ esp $=1$ \\
\hline $\mathrm{MCA}$ & Valencia & 4 & $\mathrm{NC}(3), \mathrm{HC}(1)$ & & 4 & $\begin{array}{l}\text { fra>esp }=3 \\
\text { ing }>\text { esp }=1\end{array}$ \\
\hline Media Vaca & Valencia & 1 & LIJ-NC (1) & & 1 & fra $>\operatorname{esp}=1$ \\
\hline Numa & Valencia & 4 & $\mathrm{NC}(4)$ & & 4 & ing $>$ esp $=4$ \\
\hline
\end{tabular}




\begin{tabular}{|l|l|l|l|l|l|l|}
\hline Pre-Textos & Valencia & 10 & NC (10) & & 10 & $\begin{array}{l}\text { fra }>\text { esp }=4 \\
\text { ing }>\text { esp }=4 \\
\text { ale }>\text { esp }=1 \\
\text { ita }>\text { esp }=1\end{array}$ \\
\hline Rotgle & Valencia & 3 & NC (3) & $\begin{array}{l}\text { esp }>\text { cat }=1 \\
\text { ing }>\text { cat }=1 \\
\text { fra }>\text { cat }=1\end{array}$ \\
\hline Tabarca & Valencia & 4 & LIJ-NC (2), LIJ (1), NC (1) & 4 & $\begin{array}{l}\text { ing }>\text { cat }=2 \\
\text { esp }>\text { cat }=2\end{array}$ \\
\hline Tàndem & Valencia & 23 & $\begin{array}{l}\text { LIJ (16), NC (5), DP (1), } \\
\text { LIJ-NC (1) }\end{array}$ & 23 & $\begin{array}{l}\text { eus }>\text { cat }=5 \\
\text { fra }>\text { cat }=5 \\
\text { esp }>\text { cat }=4 \\
\text { gal }>\text { cat }=4 \\
\text { ing }>\text { cat }=2 \\
\text { ale }>\text { cat }=2 \\
\text { por }>\text { cat }=1\end{array}$ \\
\hline Univ. Valencia & & & & & & \\
fra $>$ cat $=1$
\end{tabular}

Ahora sí que estamos en condiciones de conocer con claridad qué nivel de especialización han conseguido esas editoriales. Es evidente que la lengua término es siempre el catalán para las más importantes: Bromera, Eliseu Climent y Tàndem, con 91, 35 y 23 títulos respectivamente. Las demás ocupan un lugar bien discreto, con excepción si cabe de Pre-Textos, Marjal, La Máscara y Germania, con 10, 9, 8 y 7 libros respectivamente. Las grandes editoriales son también las más proclives a la diversificación de las lenguas de origen, como resulta previsible, con porcentajes no siempre similares para los pares de lenguas más productivos:

\begin{tabular}{|c|c|c|c|c|c|c|c|}
\hline & $\begin{array}{l}\text { Bromera } \\
\%\end{array}$ & $\begin{array}{l}\text { E. Climent } \\
\%\end{array}$ & $\begin{array}{l}\text { Tàndem } \\
\text { \% }\end{array}$ & $\begin{array}{l}\text { Marjal } \\
\%\end{array}$ & $\begin{array}{l}\text { Máscara } \\
\%\end{array}$ & $\begin{array}{l}\text { Pre-Textos } \\
\%\end{array}$ & $\begin{array}{l}\text { Germania } \\
\%\end{array}$ \\
\hline ing $>$ cat & 40,65 & 34,28 & 8,69 & & & & 57,14 \\
\hline fra $>$ cat & 23,07 & 17,14 & 21,73 & & & & 28,57 \\
\hline ale>cat & 5,49 & 8,54 & 8,69 & & & & 14,28 \\
\hline ita $>$ cat & 1,09 & 20,00 & & & & & \\
\hline esp $>$ cat & 2,19 & 2,85 & 17,39 & 100 & & & \\
\hline por $>$ cat & 1,09 & 2,85 & 4,34 & & & & \\
\hline eus $>$ cat & 7,69 & & 21,73 & & & & \\
\hline gal>cat & 14,28 & & 17,39 & & & & \\
\hline grc $>$ cat & & 5,71 & & & & & \\
\hline grm $>$ cat & & 2,85 & & & & & \\
\hline lat $>$ cat & & 2,85 & & & & & \\
\hline fin $>$ cat & & 2,85 & & & & & \\
\hline ára $>$ cat & 4,39 & & & & & & \\
\hline ing $>$ esp & & & & & 50,00 & 40,00 & \\
\hline fra $>$ esp & & & & & 25,00 & 40,00 & \\
\hline ale $>$ esp & & & & & 25,00 & 10,00 & \\
\hline ita>esp & & & & & & 10,00 & \\
\hline
\end{tabular}

Bromera apuesta claramente por traducir del inglés y del francés, aunque reserva un tercer lugar de privilegio para el gallego; el cuarto puesto lo ocupa el euskera, que supera incluso al alemán en volumen de traducciones. La editorial Eliseu Climent practica una pauta diferente, pues a las traducciones del inglés le siguen las del italiano (por cuyos autores se decanta); después van las del francés y las del alemán. Tàndem, por el contrario, aborda muchas traducciones del francés y del euskera (con idéntico porcentaje), seguidas por las del español y el gallego (también equilibradas). La editorial Marjal se dedica en exclusiva a traducir del 
español al catalán. Las publicaciones de La Máscara responden a porcentajes razonables para las tres primeras lenguas extranjeras de cultura. Pre-Textos traduce de forma equilibrada del inglés y del francés, e igualmente del alemán y del italiano. Por último, Germania traduce del inglés, del francés y del alemán según porcentajes también lógicos. Por otra parte, si consideramos la lengua término, la especialización de las editoriales valencianas es evidente y excluyente, pues las que publican en catalán no lo hacen en español, y viceversa.

Esta última tabla y la anterior desvelan más información relevante: la producción de seis de esas siete editoriales se justifica por su apuesta clara por la literatura infantil-juvenil y la novela-cuento. La única excepción es La Máscara, especializada más bien en temas dirigidos al gran público adulto, que solamente se traducen al español y nunca al catalán (destinatarios más jóvenes); eso queda recogido especialmente en los subgéneros LEP (literatura licenciosa, erótica y pornográfica), OG (otros géneros) y LG (literatura general).

Por otra parte, ¿qué podemos saber acerca de la apuesta por traducir a esos autores? Parece lógico pensar que el criterio general es publicar para vender, además de pretender divulgar la obra de los autores elegidos. Sin embargo, puede resultar interesante conocer el grado de compromiso de las editoriales hacia obras y autores contemporáneos en el tiempo. Ello puede deducirse a partir de un análisis de los detalles contenidos en tres de las columnas de la base de datos: la de la autoría (con la cronología vital del autor) y las que recogen las fechas de publicación del TLO (texto en lengua original) y del TLT (texto en lengua término). Con toda evidencia, la opción de publicar sin tener que abonar derechos de traducción es una de las más atractivas para las empresas editoras, pues reduce los costes de manera apreciable (en un $10 \%$ ), además de facilitar el proceso de edición ${ }^{17}$. Por el contrario, la opción de dar cobertura a autores todavía productivos o más contemporáneos supone un mayor compromiso con el lector potencial, obligando a explorar los mercados editoriales más activos. En el período 1990-2000 (siempre en contexto valenciano), la apuesta de las editoriales ha ido en la siguiente dirección:

\begin{tabular}{|l|l|l|}
\hline Editorial & $\begin{array}{l}\text { De autor } \\
\text { consagrado }\end{array}$ & $\begin{array}{l}\text { De autor } \\
\text { actual }\end{array}$ \\
\hline 7 i Mig & & 1 \\
\hline Alcodre & & 1 \\
\hline Amós Belinchón & 2 & 1 \\
\hline Bromera & 46 & 45 \\
\hline $\begin{array}{l}\text { Bromera-Univ } \\
\text { Valencia }\end{array}$ & & 1 \\
\hline Col-lectiu P.L.L. & 1 & \\
\hline Eliseu Climent & 23 & 12 \\
\hline Foro & 1 & \\
\hline Germania & 7 & \\
\hline I. E. Modernistas & 2 & 4 \\
\hline La Máscara & 4 & 9 \\
\hline Marjal & & 1 \\
\hline Mateu & & 1 \\
\hline MCA & 3 & \\
\hline Media Vaca & 1 & 3 \\
\hline Numa & 1 & 7 \\
\hline Pre-Textos & 3 & 2 \\
\hline Rotgle & 3 & 22 \\
\hline Tabarca & 2 & \\
\hline Tàndem & 1 & 110 \\
\hline Univ. Valencia & 1 & \\
\hline Total & 101 & \\
\hline
\end{tabular}


Los resultados son bastante equilibrados: se han traducido 101 autores consagrados y 110 actuales. No obstante, en las editoriales más productivas (que son las más representativas en cuanto al mercado del libro) se observan tres tendencias claras:

a) De equilibrio: Bromera mantiene una armonía casi perfecta (46/45), que es total en La Máscara (4/4).

b) De apuesta por autores consagrados: Eliseu Climent tiende a publicar obras ya conocidas en el mercado editorial (23/12), y Germania aborda exclusivamente autores consagrados (7/0).

c) De apuesta por autores contemporáneos: Tàndem invierte la tendencia anterior, optando claramente por traducir autores contemporáneos (1/22); lo mismo hace Marjal (0/9) y PreTextos (3/7).

\section{Aplicaciones proactivas de COVALT}

Aunque resulta difícil establecer prospectivas a largo plazo sobre las aplicaciones concretas de las investigaciones relacionadas con la lingüística de corpus, muchos analistas vaticinan un futuro prometedor en el campo de la traductología. Y ello por diversas razones, entre las cuales debemos destacar aquellas que hacen de los córpora instrumentos susceptibles de ser aplicados en diferentes ámbitos y para diferentes propósitos.

La contribución más evidente que puede aportar este estudio es la divulgación de la actividad traductora en el ámbito valenciano, ya que este aspecto viene presentando un claro déficit investigador. Con el método observacional expuesto anteriormente, consistente en una gestión-explotación integral de la cabecera de la base de datos COVALT, se consigue una clara sistematización del estudio analítico-descriptivo, de manera que se facilita la investigación de los fenómenos culturales relacionados con la actividad traductora, tanto de contextos contemporáneos como históricos. Además, las pautas que se han seguido pueden incentivar e impulsar un amplio abanico de posibilidades investigadoras, entre las cuales deben tenerse muy en cuenta los análisis diacrónicos literarios que apuesten por la comparación entre diversos ámbitos editoriales o sociales.

Pero el objetivo último de COVALT no es simplemente describir («cuánto se ha traducido»), sino anticiparse a las necesidades de los traductores y de los aspirantes a traductores, es decir, allanar el camino hacia «cómo traducir». De hecho, el tipo de literatura que abarca COVALT puede considerarse la ideal para la formación de traductores en nuestro contexto universitario, ya que los subgéneros dominantes en el corpus (la literatura infantil y juvenil y la novela y cuento) son los más indicados para la iniciación a la traducción literaria ${ }^{18}$. $\mathrm{Al}$ efecto, nuestro corpus pretende convertirse en un verdadero diccionario de ejemplos prácticos para los aprendices de traductores.

Puede darse ya como cierto que los estudios de corpus están contribuyendo con gran eficacia al desarrollo de la traducción automática (TA), pero también lo hacen con la traducción asistida por ordenador (TAO), ya que facilitan sobremanera la tarea de documentación, tanto de equivalencias terminológicas como de colocaciones preestablecidas por las unidades léxicas y de traducción. Por eso COVALT está creando y poniendo a punto una herramienta ad hoc que permitirá localizarlas en paralelo, de manera efectiva y rápida. Por otra parte, y habida cuenta de los intereses docentes de los miembros del equipo investigador, COVALT no ha olvidado la faceta didáctica del proyecto ${ }^{19}$. En efecto, además de la propuesta de uso del corpus para evaluar con objetividad la calidad de las traducciones, nos disponemos a desarrollar una metodología específica para enseñanza de la traducción.

En definitiva -y a nuestro entender-, la proactividad de COVALT es evidente ${ }^{20}$, máxime cuando el mercado editorial valenciano se está abriendo al exterior. Tal como recogía el 
periódico Levante-el Mercantil Valenciano el 25 de noviembre de 2004 (página 69) con motivo de la celebración del Salón del Libro (en la Biblioteca Valenciana), «un total de diez editoriales valencianas participarán en la XVIII Edición de la Feria Internacional del Libro de Guadalajara (México)-FIL 2004, que se celebrará del 27 de noviembre al 5 de diciembre». Esta política editorial, impulsada por la Associació d'Editors del País Valencià (AEPV), supone la clausura de un año (el 2004) de «fuerte estrategia de internacionalización», en el que los objetivos comerciales de los empresarios valencianos pasan por incrementar su importante presencia en el mercado latinoamericano del libro y «consolidar las incipientes relaciones con el SEP, un relevante comprador de libros destinados a las bibliotecas escolares con un presupuesto anual superior a 40 millones de dólares ${ }^{21}$. Por otra parte, los escritores y pedagogos tienen mucho que aportar al respecto, como quedó de manifiesto en el III Congreso de Literatura Infantil y Juvenil que se desarrolló en la Escuela de Magisterio Ausiàs March de la Universidad de Valencia (25-27 de noviembre de 2004). A lo largo de ese encuentro, organizado por la Associació d'Escriptors en Llengua Catalana (AELC), se debatió sobre «uno de los problemas más graves que padece la sociedad española a nivel cultural, como es el bajo índice de lectura entre su población», abogando por el fomento de la lectura infantil y juvenil. Así pues, conocer a fondo el mercado de la traducción en un contexto determinado permite corregir sus trayectorias, o actuar sobre ese medio, con objeto de suplir sus carencias y necesidades. En el caso que nos ocupa, ese conocimiento puede ser útil, por ejemplo, para adecuar los contenidos de los planes de estudios de las titulaciones de Traducción, que podrán así adaptarse a las tendencias que va marcando el mercado editorial, o para tomar decisiones acerca de la implementación de más lenguas de las que traducir, o simplemente para tomar el pulso a una sociedad a partir de la literatura que consume.

\section{Conclusión}

Como ha podido comprobarse, la gestión-explotación integral de una base de datos de literatura traducida augura importantes beneficios para la traductología. En primer lugar porque consigue relacionar la sociología de la literatura con la sociología literaria, de manera que ambas disciplinas pueden proyectar sus intereses en una sociología de la traducción con aplicaciones concretas y prácticas para sus usuarios (escritores, lectores, traductores y editoriales). En segundo lugar porque, dado un objetivo concreto (en el caso inmediato que nos ocupa, el análisis de las traducciones), esa gestión-explotación integral permite alcanzar cotas perfectamente fiables, ya que el estudio se asienta en comprobaciones empíricas. En último término, COVALT y otros córpora semejantes se revelan como el medio o denominador común capaz de aglutinar diversas y múltiples disciplinas relacionadas con las industrias de la lengua; en ese medio, su gestión-explotación integral puede considerarse el catalizador que facilita, acelera y objetiviza el empirismo de sus resultados. 


\section{NOTAS}

1. Este artículo se enmarca dentro de los siguientes proyectos de investigación: BFF2003-05422 del Ministerio de Ciencia y Tecnología, Plan Nacional de Investigación Científica, Desarrollo e Innovación Tecnológica (I+D+I), con participación de fondos FEDER de la Unión Europea, y P1 1B2003-25 de la Fundación Caixa Castelló-Bancaixa. 2. El primer proyecto, financiado por la Fundación Caixa Castelló-Bancaixa, estuvo bajo la dirección del Dr. Josep R. Guzman. El segundo, dirigido por el Dr. Josep Marco, también está financiado por la Fundación Caixa Castelló-Bancaixa. El tercero, financiado por el Ministerio de Ciencia y Tecnología, se desarrolla bajo la dirección del Dr. Joan Verdegal. 3. La misma pauta era ya seguida por el Longman Lancaster English Language Corpus (Sinclair, 1991: 23), en el cual los textos de carácter imaginativo (opuestos a los informativos) representaban un $40 \%$. De hecho, parece ser que «Tras este alto porcentaje está la idea de que los textos de ficción se leen más que los de no ficción y que, por tanto, tienen mayor influencia, tal como prueban las estadísticas de préstamo bibliotecario, de bestsellers y de lecturas recomendadas por profesores a alumnos» (Alvar y Corpas, 1994: 35).

4. Según el informe anual correspondiente al año 2003 de la Federación de Gremios de Editores de España, la Comunidad Valenciana es el cuarto territorio en volumen de edición y facturación de libros en España, detrás de Cataluña, Madrid y Andalucía respectivamente. El territorio autónomo valenciano, situado geográficamente en la costa oriental de la Península Ibérica, tiene una superficie total de $390,23 \mathrm{~km}^{2}$ y una población de 5.804 .829 habitantes (a 1 de enero de 2004). Son lenguas cooficiales el español y el catalán (con la denominación de valenciano). Si tenemos en cuenta el número de habitantes de los territorios en los que el catalán es oficial (Cataluña, Valencia, Baleares y Andorra), el catalán (con 10.700 hablantes) es la séptima lengua de la Unión Europea, por detrás del neerlandés y por delante del portugués, el griego, el sueco, el finlandés y el danés.

5. Algunos de esos peligros son expresados así por el equipo ÉCLECTIK (Marshman, 2003: 12-13): «Il convient également de se méfier de tout texte qui semble contenir beaucoup d'erreurs d'ordre linguistique. [...] Le genre du texte indique souvent son niveau de spécialisation et les qualifications d'un auteur. Par exemple, un texte d'une revue spécialisée est généralement plus approprié pour un corpus spécialisé qu'un article journalistique, qui risque d'être écrit par un non-expert pour un public qui a peu de connaissance du domaine. [...] La plupart du temps, on a tendance a éviter des textes très informels ou personnels et ceux qui sont écrits par des membres du grand public, qui risquent de contenir du vocabulaire et de la phraséologie inexacts».

6. No se han perdido de vista aquí aportaciones tan importantes como las de R. Escarpit (1958) y de J.-P. Sartre (1964). En el contexto catalán, los antecedentes prácticos de este estudio se hallan en tres artículos de J. Verdegal (1997, 1999a y 1999b). En el ámbito gallego, la apuesta decidida del corpus TECTRA queda reflejada en la siguiente frase de Álvarez Lugrís (2001: 190): «En el caso de las traducciones al gallego, de este tipo de textos podemos extraer más conclusiones y más fiables sobre pautas de comportamiento traductivo -e insistimos, no sólo literario-, criterios de traducción, políticas editoriales, etc., que de ningún otro tipo de texto».

7. El espacio disponible nos impide recoger aquí esa tabla. Una versión simplificada se encuentra en <http://www.covalt.uji.es>, donde hemos preferido obviar la codificación, a fin de hacer más legibles las cabeceras. Nos hemos basado en operaciones de etiquetado ya habituales entre los estudiosos de corpus (Vivaldi y otros, 1996; Álvarez Lugrís, 2001).

8. En COVALT, la columna 9 es de relativa utilidad para el primero de los aspectos, ya que la etapa estudiada es demasiado corta (1990-2000) y no se recoge la primera traducción de cada original, sino sólo la primera aparecida en esos años. Sin embargo, sí que es útil para desvelar las políticas editoriales sobre la traducción de obras consagradas o recientes. Por otro lado, en el ámbito de la sociología literaria, esta misma columna serviría para analizar los comportamientos receptivos del lector virtual, tal como los describe Iser (1979: 291): si la traducción es contemporánea a la obra original, el lector de la traducción participa más directamente del sistema significativo de la obra y vive la novedad del texto; por el contrario, si existe mucha distancia temporal entre obra y traducción, la lectura pasa a ser más contemplativa, de forma que el lector no la vive como experiencia.

9. Una de las novelas (la número 6) ha merecido dos traducciones diferentes. No se consideran en este bloque las reediciones, aunque podrían servir para conocer los posibles éxitos de ventas.

10. Se comparte igualmente traductor en otros cuatro casos, pero los dobletes han sido eliminados de COVALT por participar en ellos editoriales catalanas (no adscritas, por tanto, al ámbito valenciano).

11. Hay que añadir otros cinco traductores desconocidos, cuyo nombre no aparece ni en las bates de datos del Ministerio de Cultura ni en las páginas de crédito de los libros. Con objeto de desvelar esos nombres, se ha abierto un proceso de documentación (mediante consultas a las editoriales) que todavía no se ha cerrado. Aunque las causas del anonimato pueden ser diversas, se adivina cierta intencionalidad, ya que casi todas esas obras pueden considerarse de temática escabrosa.

12. Una de ellas, sin embargo, es del autor alemán Rudolph Eric Raspe (1737-1794), cuya traducción al catalán se ha realizado a partir de la versión inglesa The Surprising Adventures of Baron Munchausen. 
13. Contamos aquí con la traducción del libro en euskera Tigre ehizan del escritor Aingeru Epalza, ya que se ha realizado a partir de su versión en español (Cazador de tigres).

14. Dos de ellas utilizan el gallego como lengua puente (Palabras màxicas, publicada por dos editoriales), ya que el texto original es asturiano, de Pablo Rafael Prestifilippo Lajud-Cura.

15. Las fichas catalográficas del ISBN no son del todo fiables. Es discutible, por ejemplo, que la novela autobiográfica de Luise Rinser Dietari de presó pertenezca al subgénero del derecho penal, o que Las mil y una noches traducidas por Vicente Blasco Ibáñez sean historia y crítica.

16. Parece ser que las editoriales valencianas están especializadas en ciertas áreas temáticas. Además, por su volumen de producción puede deducirse que algunas de ellas se dedican a la edición de manera ocasional. Según estudios de la Federación de Gremios de Editores de España (2004), se trata de editoriales pequeñas, cuya media de empleados es de siete por empresa.

17. La Ley de Propiedad Intelectual en vigor (RD legislativo 1/1996) dispone en su artículo 26 que «Los derechos de explotación de la obra durarán toda la vida del autor y setenta años después de su muerte [...]». Además de los derechos de autor, que en las editoriales comerciales suelen ser de un $8 \%$, existen otros derechos, como los de ediciones recientes.

18. En el caso de la titulación de Traducción e Interpretación de la Universitat Jaume I, todos los estudiantes traducen del inglés (lengua B) al español o al catalán (según sea su lengua A: A1 o A2). Sin embargo, las lenguas C (alemán o

francés) no disponen en el plan de estudios de asignaturas de traducción literaria (sólo se imparte traducción general), por lo que COVALT resulta de gran utilidad como herramienta de iniciación a esa especialidad traductora.

19. Las publicaciones con aplicaciones didácticas de los córpora se suceden con bastante celeridad, hasta el punto de que resultaría arduo recogerlas aquí. Sin embargo, algunas de las más recientes son las aportaciones de López Chollet (2001), Vargas (2002), Zanettin y otros (2003) y Bernardini (2004).

20. En última instancia, las vocaciones de COVALT se ampliarán (en la medida en que lo haga el propio equipo investigador) a los diversos usos de los córpora de traducción, que, según recoge Corpas (2001: 159-164), pueden ser: a) la comparación interlingüística (con especial atención a la determinación de la unidad de traducción), b) la caracterización de la lengua traducida (análisis del comportamiento traductor), c) la traducción automática, d) las bases de datos para la traducción asistida por ordenador y e) la docencia y aplicaciones didácticas.

21. Es de suponer que las editoriales echarán mano de muchos de los títulos publicados los años anteriores, que deberán entrar nuevamente en un proceso de encargo de traducciones al español, por lo que se prevé un significativo aumento de contratos de traducción. Esta evolución deberá tenerse en cuenta desde el observatorio COVALT, entre otras cosas porque puede inclinar la balanza a favor de la traducción al español, ahora minoritaria.

\section{REFERENCIAS}

Alvar EzQuerRA, M. y G. CORPAS PASTOR (1994): «Criterios de diseño para la creación de córpora», M. Alvar Ezquerra y J. A. Villena Ponsoda (coords.): Estudios para un corpus del español, Málaga, Universidad de Málaga, p. 31-40.

ÁlvarEZ LugRís, A. (2001): TECTRA: teoría y práctica de las investigaciones con corpus en el marco de los Estudios de Traducción, Trans: revista de traductología, 5, p. 185-204.

BERNARDINI, S. (2004): «Corpora in the classroom», en John Sinclair (ed.): How to use corpora in language teaching, Amsterdam, Benjamins, p. 15-36.

CORPAS PASTOR, G. (2001): «Compilación de un corpus ad hoc para la enseñanza de la traducción inversa especializada», Trans: revista de traductología, 5, p. 155-184.

ESCARPIT, R. (1958): Sociologie de la littérature, París, PUF.

FEDERACIÓN DE GREMIOS DE EDITORES DE ESPAÑA (2004): Comercio interior del libro en España. 2003, Madrid, FGEE.

GILE, D. (1998): «Observational Studies and Experimental Studies in the Investigation of Conference Interpreting», Target, 10/1, p. 69-93.

GuZman, J. R. (1995): Les teories de la recepció literària, Valencia, Universitat de València, Secretariat de Publicacions de la Universitat d'Alacant, Publicacions de la Universitat Jaume I.

ISER, W. (1979): «La fiction en effet», Poétique 39, p. 275-298.

LEENHARDT, J. (2003): «Sociologie de la littérature», <http://www.arfe-cursus.com>, p. 1-11.

LEVANTE-El MERCANTIL VALENCIANO (25-12-2004): «Las editoriales valencianas abren mercado en América», «Escritores y pedagogos analizan el futuro de la literatura infantil», p. 69. 
LóPez ChOllet, M. J. (2001): «Utilización de corpus en la formación del traductor», C. Valero Garcés e I. de la Cruz Cabanillas (eds.): Traducción y nuevas tecnologías. Herramientas auxiliares del traductor, Alcalá de Henares, Universidad de Alcalá de Henares, p. 317-325.

MARCO, J., Verdegal, J. y A. HURTADO (1999): «La traducción literaria», A. Hurtado Albir (Dir.): Enseñar a traducir. Metodología en la formación de traductores e intérpretes, Madrid, Edelsa, p. 167-181.

MARSHMAN, E. (2003): «Construction et gestion des corpus: Résumé et essai d'uniformisation du processus pour la terminologie», < http://www.olst.umontreal.ca/pdf/terminotique/corpusentermino.pdf>.

Ministerio De Cultura (1980-2000): Bibliotecas Públicas del Estado (Catálogos), Madrid, $<$ http://www.mcu.es/cgi-bin/cbpe_b $>$.

MiNISTERIO DE CUlTURA (1980-2000): Agencia Española del ISBN, Madrid, $<$ http://www.mcu.es/bases/spa/isbn/ISBN.html>.

Sinclair, J. (1991): Corpus, Concordance, Collocation, Oxford, Oxford University Press.

SARTRE, J.-P. (1964): Qu'est-ce que la littérature?, París, Gallimard.

VARGAS SIERRA, C. (2002): «Utilización de los programas de concordancias en la traducción especializada», P. Hernúñez y L. González (eds.): El español, lengua de traducción, Actas del I Congreso Internacional, Almagro, Comisión Europea/Agencia EFE, p. 468-483.

VERDEGAL, J. (1997): «La traducció de la narrativa francesa al català: un factor de la normalització», C. Benoit, F. Carbó, D. Jiménez y V. Simbor (eds.): Les literatures catalana i francesa al llarg del segle XX / Les littératures catalane et française au XXème siècle, Barcelona, Publicacions de l'Abadia de Montserrat, p. 385-407.

VERDEGAL, J. (1999a): «La traduction des romans catalans en français: le rôle des intermédiaires», Translatio. Nouvelles de la F.I.T., XVIII, 2, p. 176-216.

VERDEGAL, J. (1999b): «La réciprocité de la traduction de romans (contexte français-catalan, catalan-français): vers un déséquilibre harmonieux», Traduire, 180. Lettres et terminologie, p. 7-31.

Vivaldi, J., De YZaguirRe, Ll., SolÉ, X. y M. T. CABRÉ (1996): Marcatge Estructural i Morfosintàctic del Corpus Tècnic amb l'estàndard SGML. Sèrie Informes, 1, Barcelona, Universitat Pompeu Fabra. Institut Universitari de Lingüística Aplicada.

WARNING, R. (ed.)(1989): Estética de la recepción, Madrid, Visor.

Zanettin, F., Bernardini, S. y D. Stewart (eds.)(2003): Corpora in translator education, Manchester, St. Jerome. 